\begin{tabular}{|c|c|}
\hline Title & Homogenization Method Based on Cauer Circuit via U nit Cell A pproach \\
\hline Author(s) & Hiruma, Shingo; Igarashi, Hajime \\
\hline Citation & $\begin{array}{l}\text { IEEE transactions on magnetics, 56(2), 1-5 } \\
\text { https://doi.org/10.1109/T MA G.2019.2946402 }\end{array}$ \\
\hline Issue Date & $2020-02$ \\
\hline Doc URL & http://hdl.handle.net/2115/77539 \\
\hline Rights & $\begin{array}{l}\text { (c) } 2020 \text { IEEE. Personal use of this material is permitted. Permission from IEEE must be obtained for all other uses, in } \\
\text { any current or future media, including reprinting/republishing this material for advertising or promotional purposes, } \\
\text { creating new collective works, for resale or redistribution to servers or lists, or reuse of any copyrighted component of } \\
\text { this work in other works. }\end{array}$ \\
\hline Tyре & article (author version) \\
\hline File Information & hiruma_homogenization_based_on_Cauer.pdf \\
\hline
\end{tabular}

Instructions for use 


\title{
Homogenization Method Based on Cauer Circuit via Unit Cell Approach
}

\author{
Shingo Hiruma ${ }^{1,2}$, Hajime Igarashi ${ }^{1}$, IEEE Member \\ ${ }^{1}$ Graduate School of Information Science and Technology, Hokkaido University, Hokkaido 060-0814, Japan \\ ${ }^{2}$ Research Fellow of Japan Society for the Promotion of Science (JSPS), Tokyo 102-0083, Japan
}

\begin{abstract}
This paper proposes a novel homogenization method based on the unit cell approach which provides the continued fraction and, equivalently, Cauer circuit representation of the complex permeability of fine structure materials. The proposed method makes it possible to perform the homogenization analysis in time domain. It is shown that the proposed method provides more accurate resistance factor in comparison with the Dowell method and other classical methods.
\end{abstract}

Index Terms - Complex permeability, Homogenization method, Proximity effect Resistance factor, Unit cell, Dowell method.

\section{INTRODUCTION}

$\mathrm{T}$ HE FINITE ELEMENT METHOD (FEM) has been widely used as the numerical method to analyze the electric machines and devices. In the FE analysis of electric apparatus, we sometimes encounter the situation that the fine structured components such as the litz wire and soft magnetic composite have to be modeled. In this case, the number of the finite elements could be enormous when we subdivide them to finite elements smaller than the skin depth. This problem is referred to as the multi-scale problem, which cannot be effectively treated by the conventional FEM.

The homogenization method has been proposed to deal with the multi-scale problem [1]-[4]. In this method, a fine structure component is modeled as a uniform material with the complex permeability, which is a complex function of frequency whose real and imaginary parts represent the permeability decreased by the diamagnetization due to the eddy currents and eddy current losses, respectively. Using the complex permeability, we can evaluate the eddy current losses through the coarse FE model of the homogenized material without fine discretization.

In this paper, we propose a new method to formulate the complex permeability in the form of the continued fraction which is equivalent to the Cauer circuit representation. The advantages of the present method are: (a) the complex permeability expressed by continued fraction can be obtained from 2D/3D unit cells including arbitrary-shaped material, (b) since the Cauer circuit is directly synthesized from the continued fraction [5], time-domain analysis is readily performed, (c) the equivalent circuit of the winding considering the eddy current losses can be obtained by using the complex permeability, which is more accurate than the method of Dowell [6] and other analytical methods [7] in high frequency domain.

Manuscript received April 1, 2015; revised May 15, 2015 and June 1, 2015; accepted July 1, 2015. Date of publication July 10, 2015; date of current version July 31, 2015. (Dates will be inserted by IEEE; "published" is the date

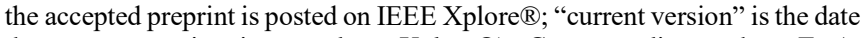
the typeset version is posted on Xplore $\left.{ }^{\circledR}\right)$. Corresponding author: F. A. Author (e-mail: f.author@nist.gov).

Digital Object Identifier (inserted by IEEE).

\section{FORMULATION}

\section{A. Homogenization based on unit cell}

The proposed method is based on the unit cell approach in which the fine structure of interest is assumed to be composed of spatially-periodic unit cells. Let us consider domain $\Omega_{h}$ which is composed of the unit cells $\Omega_{u}$ containing conductive materials and air region, as shown in Fig. 1 , where $\Gamma_{D}, \Gamma_{N}$ denote

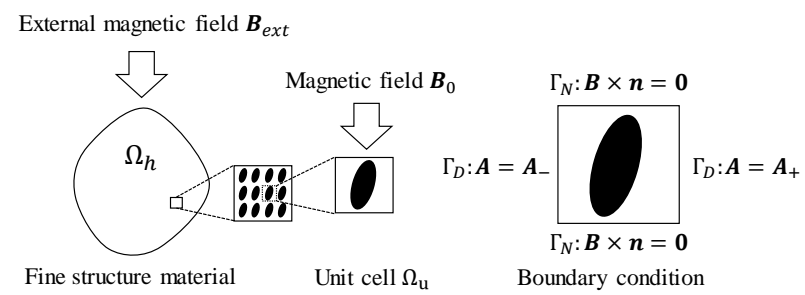

Fig. 1 Unit cell

the Dirichlet and Neumann boundaries. It is assumed that the unit cells are immersed in a uniform time-harmonic magnetic field $\boldsymbol{B}_{0}$. The boundary conditions

$$
\boldsymbol{B} \times \boldsymbol{n}=\mathbf{0} \text { on } \Gamma_{N}, \quad \boldsymbol{A}=\boldsymbol{A}_{+}, \boldsymbol{A}_{-} \text {on } \Gamma_{D}
$$

are imposed on the boundary of $\Omega_{\mathrm{u}}$ where $\boldsymbol{n}$ is the outer normal unit vector on boundary $\Gamma_{N}$. The quasi-static Maxwell equations

$$
\operatorname{rot} \boldsymbol{H}=\sigma \boldsymbol{E}, \quad \operatorname{rot} \boldsymbol{E}=-\mathrm{j} \omega \boldsymbol{B}
$$

are solved in $\Omega_{u}$ where $\sigma, \mathrm{j}, \omega$ represent the conductivity, imaginary unit, and angular frequency, respectively. Solving (2), the complex permeability $\langle\dot{\mu}\rangle$ is obtained from

$$
\langle\dot{\mu}\rangle=\frac{\int_{\Omega_{u}}\left|\boldsymbol{B}_{0}\right|^{2} \mathrm{~d} \Omega}{\int_{\Omega_{u}} \frac{|\boldsymbol{B}|^{2}}{\mu} \mathrm{d} \Omega-\frac{1}{\mathrm{j} \omega} \int_{\Omega_{u}} \sigma|\boldsymbol{E}|^{2} \mathrm{~d} \Omega}
$$

where $\mu$ is the permeability. Eq. (3) is derived from the requirement that the energy in the original cell is equal to that in the homogenized unit cell [2].

\section{B. Discretization of complex permeability}

To represent the complex permeability in an explicit form, we make FE discretization of (3). To do so, (2) is discretized by FEM to obtain 


$$
(K+s N) \boldsymbol{x}=\boldsymbol{b}\left|\boldsymbol{B}_{\mathbf{0}}\right|
$$

where $K, N \in \mathbb{R}^{n \times n}, \boldsymbol{x} \in \mathbb{C}^{n}, \boldsymbol{b} \in \mathbb{R}^{n}, s \in \mathbb{C}$ are the FE matrices, unknown and right-handed vectors, and complex frequency $\mathrm{j} \omega$, respectively. From the energy conservation law which is derived from (2), we have

$$
s \int_{\Omega_{u}} \frac{|\boldsymbol{B}|^{2}}{\mu} \mathrm{d} \Omega+\int_{\Omega_{u}} \sigma|\boldsymbol{E}|^{2} \mathrm{~d} \Omega=-\int_{\partial \Omega_{u}} \boldsymbol{E} \times \boldsymbol{H}^{*} \cdot \boldsymbol{n} \mathrm{d} S
$$

where $*$ denotes the conjugate operator. The right-handed side of (5), which represents the surface integral of the Poynting vector, is discretized by FEM to obtain

$$
\begin{aligned}
-\int_{\partial \Omega_{u}} \boldsymbol{E} \times \boldsymbol{H}^{*} \mathrm{~d} \Omega & =s \boldsymbol{c}^{\mathrm{T}} \boldsymbol{x}^{*}\left|\boldsymbol{B}_{0}\right| \\
& =s \boldsymbol{c}^{\mathrm{T}}\left(K+s^{*} N\right)^{-1} \boldsymbol{b}\left|\boldsymbol{B}_{0}\right|^{2} .
\end{aligned}
$$

Inserting (5) into (3), $\langle\dot{\mu}\rangle$ is expressed in the discretized form

$$
\langle\dot{\mu}\rangle=\frac{\int_{\Omega_{u}}\left|\boldsymbol{B}_{0}\right|^{2} \mathrm{~d} \Omega}{-\frac{1}{S} \int_{\partial \Omega_{u}} \boldsymbol{E}^{*} \times \boldsymbol{H} \cdot \boldsymbol{n} \mathrm{d} S}=\frac{V}{\boldsymbol{c}^{\mathrm{T}}(K+s N)^{-1} \boldsymbol{b}} .
$$

where $V=\int_{\Omega_{u}} d \Omega$ denotes the volume of the unit cell.

\section{Cauer representation of complex permeability}

The Cauer representation of the complex permeability is effective especially when performing the time-domain analysis and considering nonlinearity of the homogenized material. For this reason, we derive the Cauer representation of (7). By expanding the denominator of (7), we obtain

$$
\begin{aligned}
\boldsymbol{c}^{\mathrm{T}}(K+s N)^{-1} \boldsymbol{b} & =\boldsymbol{l}^{\mathrm{T}}(I-s A)^{-1} \boldsymbol{r} \\
& =\boldsymbol{l}^{\mathrm{T}} \boldsymbol{r}+s \boldsymbol{l}^{\mathrm{T}} A \boldsymbol{r}+s^{2} \boldsymbol{l}^{\mathrm{T}} A^{2} \boldsymbol{r}+\cdots \\
& =\boldsymbol{l}^{\mathrm{T}} \boldsymbol{r}+s \boldsymbol{l}^{\mathrm{T}}(I-s A)^{-1} A \boldsymbol{r}
\end{aligned}
$$

where $\boldsymbol{l}=\boldsymbol{c}, \boldsymbol{r}=K^{-1} \boldsymbol{b}, A=-K^{-1} N$. The first and second terms in (8) represent the DC inductance and frequency dependent term. By applying the Cauer via the Lanczos (CVL) algorithm [8] summarized in Appendix A, developed by the authors, to the second term of (8), we obtain the finite continued fraction expression as follows:

$$
\begin{aligned}
\langle\dot{\mu}\rangle & =\frac{1}{\frac{1}{\kappa_{0}}+\frac{1}{\frac{1}{s \kappa_{1}}+\frac{1}{\frac{1}{\kappa_{2}}+\frac{1}{\ddots}}}} \\
& \triangleq\left[0 ; \frac{1}{\kappa_{0}}, \frac{1}{s \kappa_{1}}, \frac{1}{\kappa_{2}}, \cdots, \frac{1}{\kappa_{2 q-2}}, \frac{1}{s \kappa_{2 q-1}}\right]
\end{aligned}
$$

The constants $\kappa_{n}$ can be obtained by the CVL algorithm. Since the continued fraction corresponds to the input impedance of the Cauer ladder circuit which is composed of a finite number of lumped elements, the complex permeability is now represented by the Cauer circuit. Therefore, it can be used in homogenized FE analysis in frequency as well as time domains. It is remarked that the response computed by the proposed method might have numerical errors for sharp transitions due to the truncation error in the Cauer circuit as well as FEM. The theoretical analysis of these errors remains as a future task.

\section{NUMERICAL RESULTS}

\section{A. 2-D unit cell model}

The proposed method is applied to the analysis of a multiturn coil composed of the unit cells, $w \times w$, each of which includes a cylindrical conductor of radius $a$, as shown in Fig.2. The relative permeability and conductivity of the conductor are assumed to be 1 and $5.76 \times 10^{7} \mathrm{~S} / \mathrm{m}$. The complex permeability is computed by the proposed method and semianalytical method based on the extended Ollendorff formula [3] The results are plotted in Fig.2. The horizontal axis denotes the radius of the wire $a$ normalized by the skin depth $\delta$. We consider five different values of the ratio $2 a / w$. The continued fraction is truncated at $q=5$. We can see the results obtained by the proposed method is in good agreement with those obtained by the semi-analytical method.

The proposed method can treat the unit cell including arbitrary-shaped conductors which cannot be accurately modeled by the semi-analytical method. The complex permeability of a multi-turn rectangular coil computed by the proposed method is plotted against frequency in Fig.3, where the complex permeability is anisotropic due to the shape of the unit cell, which can be obtained by applying the uniform magnetic fields parallel to $\mathrm{x}$ and $\mathrm{y}$ axes independently. The values in the continued fraction are shown in Table I. The complex permeability of the rectangular coil can also be computed by the method proposed in [3] which has to introduce the generalized demagnetization factor $N$. In contrast, the

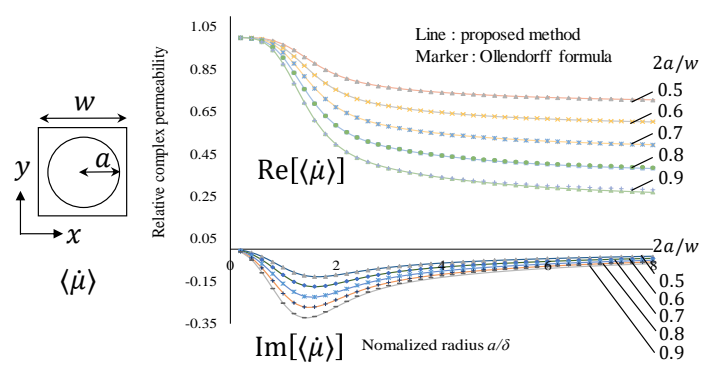

Fig.2 Frequency characteristics of complex permeability for a multi-turn round coil with the unit cell shown left.
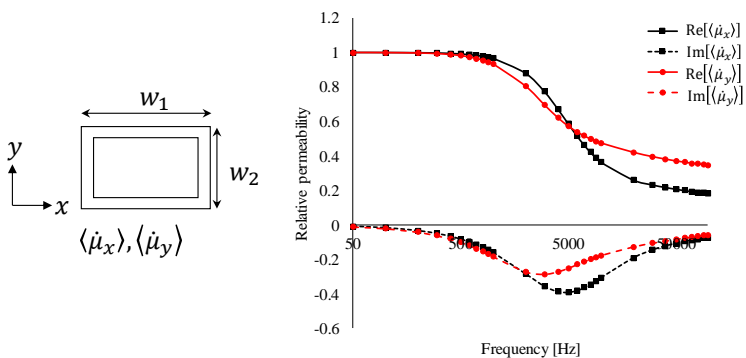

Fig.3 Frequency characteristics of complex permeability in $x$ and $y$ directions for a multi-turn rectangular coil with the unit cell shown left. TABLE I VALUES IN CONTINUED FRACTION OBTAINED BY PROPOSED METHOD.

\begin{tabular}{ccccc} 
& \multicolumn{2}{c}{$\left\langle\dot{\mu}_{r x}\right\rangle$} & \multicolumn{2}{c}{$\left\langle\dot{\mu}_{r y}\right\rangle$} \\
\hline$i$ & $\kappa_{2 i-2}$ & $\kappa_{2 i-1}$ & $\kappa_{2 i-2}$ & $\kappa_{2 i-1}$ \\
\hline 1 & $1.000 \times 10^{0}$ & $4.223 \times 10^{-4}$ & $1.000 \times 10^{0}$ & $2.530 \times 10^{-4}$ \\
2 & $4.441 \times 10^{-1}$ & $5.364 \times 10^{-5}$ & $7.369 \times 10^{-1}$ & $3.213 \times 10^{-5}$ \\
3 & $8.277 \times 10^{-1}$ & $1.030 \times 10^{-5}$ & $1.381 \times 10^{0}$ & $5.780 \times 10^{-6}$ \\
4 & $1.732 \times 10^{0}$ & $2.826 \times 10^{-6}$ & $-7.016 \times 10^{0}$ & $-1.891 \times 10^{-4}$ \\
5 & $3.286 \times 10^{0}$ & $8.729 \times 10^{-7}$ & $2.035 \times 10^{0}$ & $1.668 \times 10^{-6}$ \\
\hline \hline
\end{tabular}


proposed method can directly provide the complex permeability without introducing $N$.

\section{B. Comparison with classical methods}

To show the merit of the Cauer representation, the complex permeability is applied to the evaluation of the eddy current losses due to the proximity effect in a copper winding. Evaluation of the proximity loss is important for design of highfrequency (HF) transformers, resonant inductors, motor windings, and so on to improve the efficiency [6], [7], [9].

Let us consider the copper winding, $z_{a} \times \mathrm{z}_{t}$ turns, in a slot whose permeability is assumed infinite as shown in Fig. 4. This assumption is necessary not for the proposed method but for the classical method mentioned below. The width and height of the subconductors are $b_{\mathrm{c} 0}$ and $h_{c 0}$. Alternative current $I$ parallel to $z$-axis flows in each conductor. The homogenized copper winding is also shown in Fig. 4 where $\Omega_{h}$ denotes the homogenized winding. The width and height of the homogenized winding are $b_{\mathrm{c}}=z_{a} b_{\text {unit }}$ and $h_{\mathrm{c}}=z_{t} h_{\text {unit }}$ where $b_{\text {unit }}$ and $h_{\text {unit }}$ are the width and height of the unit cell. The current density and net current in the homogenized domain are $J$ and $\int_{\Omega_{h}} J \mathrm{dS}=z_{a} z_{t} I$.

The average resistance factor of the winding at frequency $\omega$ which is defined by $k=R_{\mathrm{AC}} / R_{\mathrm{DC}}$ can be evaluated from [10]

$$
\begin{aligned}
& k=\xi \frac{\sinh 2 \xi+\sin 2 \xi}{\cosh 2 \xi-\cos 2 \xi}+2 \xi \frac{z_{t}^{2}-1}{3} \frac{\sinh \xi-\sin \xi}{\cosh \xi+\cos \xi} \\
& \xi=h_{c 0} \sqrt{\frac{1}{2} \omega \sigma \mu \frac{z_{a} b_{c 0}}{b}}
\end{aligned}
$$

where $\sigma, \mu$ represents the conductivity and permeability of the conductors. This classical approach is widely used in the design of electric apparatuses [9]. Note that this approach, based on the method of Dowell and other analytical methods, can be inaccurate when its main assumptions that the magnetic field is one-dimensional and the eddy currents in the subconductors have no influence on the global field do not hold.

Though the proposed method is available for $2 \mathrm{D}$ and $3 \mathrm{D}$ models, it is applied to the simple model shown in Fig.4 for comparison. Using the proposed method, the impedance and resistance factor of the homogenized winding are expressed as

$$
\begin{aligned}
& \dot{Z}=z_{a} z_{t} R_{\mathrm{DC}}+\mathrm{j} \omega\langle\dot{\mu}\rangle \frac{h_{c} b_{c} l z_{a}^{2} z_{t}^{2}}{3 b^{2}} \\
& k=\frac{\operatorname{Re}[\dot{Z}]}{z_{a} z_{t} R_{\mathrm{DC}}}=1-\operatorname{Im}[\langle\dot{\mu}\rangle] \frac{\omega h_{c} b_{c} l z_{a} z_{t}}{3 b^{2} R_{D C}}
\end{aligned}
$$

where $l$ denotes the length of the subconductor and $R_{D C}=$ $l / \sigma h_{c 0} b_{c 0}$ represents the DC resistance of a subconductor. Eq. (11a) is obtained by solving the 1D equation for magnetostatic field while the 2D structure is reflected in $\langle\dot{\mu}\rangle$ (see Appendix B). Note that the neglected effects in (10) are considered in (11).

To validate the proposed method, we consider the winding as shown in Fig. 5 for $h_{c 0}=2 \mathrm{~mm}, b_{c 0}=10 \mathrm{~mm}, b_{c}=14 \mathrm{~mm}$, $z_{a}=1, z_{t}=12, h_{\text {unit }}=2.38 \mathrm{~mm}, b_{\text {unit }}=12 \mathrm{~mm}, \sigma=$
$5.76 \times 10^{7} \mathrm{~S} / \mathrm{m}$. The resistance factors are computed by (10), (11b), and FEM as shown in Fig.5. In the FE analysis, the relative permeability of the core is assumed to be 10000 instead of infinite permeability. When frequency is relatively low, the three results are in good agreement. However, the conventional resistance factor evaluated from (10) has discrepancies from the other two results as frequency increases.

In addition, the equivalent circuit of the winding can be developed by (11a) because the complex permeability is represented by the continued fraction. The impedance of the winding can be written as follows:

$$
\dot{Z}=\left[z_{a} z_{t} R_{\mathrm{DC}} ; \frac{1}{s c \kappa_{0}}, \frac{c}{\kappa_{1}}, \cdots, \frac{1}{s c \kappa_{2 q-2}}, \frac{c}{\kappa_{2 q-1}}\right]
$$

where $c=h_{c} b_{c} l z_{a}^{2} z_{t}^{2} / 3 b^{2}$. The equivalent circuit is shown in Fig. 6. Considering more general cases such as interleaved transformer winding, the equivalent circuit can be obtained by using magnetomotive force (m.m.f.) diagram which is utilized in the method of Dowell. Analyzing the equivalent circuit by the circuit simulator, we can evaluate the eddy current losses even when the non-sinusoidal current flows in the conductors.

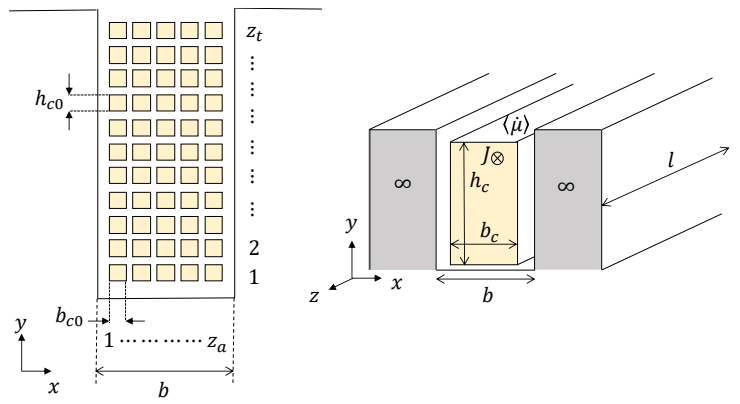

Fig. 4 Copper winding in a slot whose permeability is infinite. All conductors are connected in series. Left and right figures show the original and homogenized windings.

validation model homogenized model 300
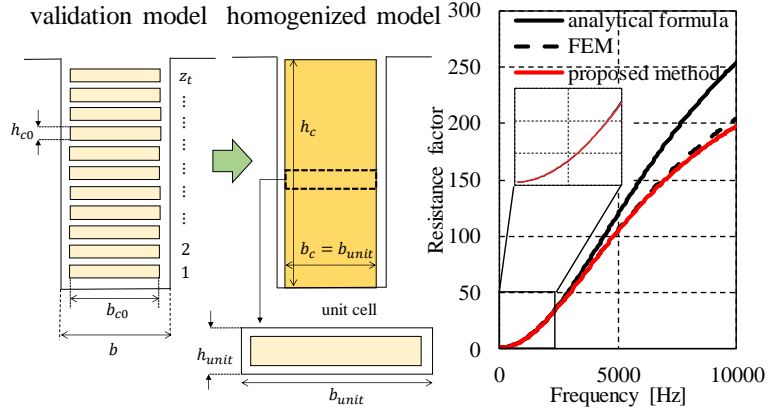

Fig. 5 Validation model and frequency characteristic of the resistance factor.

$\xi_{L_{1}} \xi_{L_{2}} \xi_{L_{3}}^{R_{1} z_{t} R_{D C}} \xi_{L_{4}}^{R_{2}}$

$$
L_{i}=c \kappa_{2 i-2} \quad R_{i}=\frac{c}{\kappa_{2 i-1}}
$$

Fig. 6 Equivalent Cauer circuit of winding considering proximity effect.

Moreover, the magnetic saturation in the core can be considered by connecting the nonlinear inductor in series with the circuit.

\section{CONCLUSION}

A new homogenization method based on the unit cell 
approach has been proposed. This method allows us to obtain the complex permeability represented by the Cauer circuit for any unit cell. The obtained complex permeability is more accurate than the widely-used classical method developed by Dowell and other researchers because the assumptions in the classical method can be relaxed in the proposed method. Since the complex permeability is represented by the equivalent circuit, we can readily perform time domain analysis.

\section{APPENDIX A}

Using the CVL algorithm [8] which is a variant of Padé approximation via Lanczos (PVL) [11], the transfer function can be approximated by the finite continued fraction.

Let us consider the linear system

$$
\begin{aligned}
& (K+s N) \boldsymbol{x}=\boldsymbol{b} u, \\
& y=\boldsymbol{c}^{\mathrm{T}} \boldsymbol{x}
\end{aligned}
$$

where $K, N \in \mathbb{R}^{n \times n}, \boldsymbol{b}, \boldsymbol{c} \in \mathbb{R}^{n}$. The response of the system can be expressed by the transfer function

$$
\mathcal{H}(s)=\frac{y}{u}=\boldsymbol{c}^{\mathrm{T}}(K+s N)^{-1} \boldsymbol{b} .
$$

It is often difficult to compute the transfer function directly when $n$ is large. In this case, it is effective to approximate the transfer function by the rational function. The CVL algorithm represented in the pseudo code below allows approximating the transfer function by the finite continued fraction.

\section{CVL algorithm}

0) Set

Compute

$$
\begin{aligned}
& \boldsymbol{u}_{0}=K^{-1} \boldsymbol{b}, \quad \boldsymbol{u}_{0}^{\star}=\boldsymbol{c}, \\
& \kappa_{0}=\left(\boldsymbol{u}_{0}^{\star}, \boldsymbol{u}_{0}\right), \quad A=K^{-1} N .
\end{aligned}
$$

For $n=1,2, \cdots, q$ do:

$$
\begin{aligned}
& \boldsymbol{v}_{0}=\boldsymbol{u}_{0} / \kappa_{0}, \quad \boldsymbol{v}_{0}^{\star}=\boldsymbol{u}_{0}^{\star} / \kappa_{0}, \\
& \kappa_{1}=\left(\boldsymbol{v}_{0}^{\star}, A \boldsymbol{v}_{0}\right) .
\end{aligned}
$$

1) Compute

$$
\begin{aligned}
\boldsymbol{u}_{n} & =\boldsymbol{u}_{n-1}-A \boldsymbol{v}_{n-1} / \kappa_{2 n-1}, \\
\boldsymbol{u}_{n}^{\star} & =\boldsymbol{u}_{n-1}^{\star}-A^{\mathrm{T}} \boldsymbol{v}_{n-1}^{\star} / \kappa_{2 n-1}, \\
\kappa_{2 n} & =\left(\boldsymbol{u}_{n}^{\star}, \boldsymbol{u}_{n}\right) .
\end{aligned}
$$

2) Compute

$$
\begin{aligned}
\boldsymbol{v}_{n} & =\boldsymbol{v}_{n-1}+\boldsymbol{u}_{n} / \kappa_{2 n}, \\
\boldsymbol{v}_{n}^{\star} & =\boldsymbol{v}_{n-1}^{\star}+\boldsymbol{u}_{n}^{\star} / \kappa_{2 n}, \\
\kappa_{2 n+1} & =\left(\boldsymbol{v}_{n}^{\star}, A \boldsymbol{v}_{n}\right) .
\end{aligned}
$$

In this algorithm, the generated vectors $\boldsymbol{u}_{i}, \boldsymbol{u}_{i}^{\star}, \boldsymbol{v}_{i}, \boldsymbol{v}_{i}^{\star}$ satisfy the following bi-orthogonal conditions.

$$
\left(\boldsymbol{u}_{i}^{\star}, \boldsymbol{u}_{j}\right)=\kappa_{2 i} \delta_{i j}, \quad\left(\boldsymbol{v}_{i}^{\star}, A \boldsymbol{v}_{j}\right)=\kappa_{2 \mathrm{i}+1} \delta_{i j}
$$

where $\delta_{i j}$ denotes Kronecker's delta. Application of CVL to (A2) results in the continued fraction as follows:

$$
\begin{aligned}
\mathcal{H}(s) & =\boldsymbol{c}^{\mathrm{T}}(K+s N)^{-1} \boldsymbol{b} \\
& =\left[0 ; \frac{1}{\kappa_{0}}, \frac{1}{s \kappa_{1}}, \cdots, \frac{1}{\kappa_{2 q-1}}, \frac{1}{s \kappa_{2 q}}\right]+O\left(s^{2 q}\right)
\end{aligned}
$$

\section{APPENDIX B}

Let us consider the infinite long homogenized winding immersed in a slot, as shown in Fig. 4. If the permeability of the iron is assumed infinite, the magnetic field $\boldsymbol{H}$ have only the $x$ component across the conductor. Applying Ampère's law, we obtain

$$
-\frac{\partial H_{x}}{\partial y}=\frac{b_{c}}{b} J .
$$

Since the current density is uniform in the homogenized domain, the partial differential equation can be integrated,

$$
H_{x}=-\frac{b_{c} J}{b} y+\text { const } \text {. }
$$

The integration constant can be determined from the boundary condition $H_{x}=0$ at $y=0$. As a result, we find

$$
\text { const }=0 \text {. }
$$

The impedance in the homogenized domain can be obtained as

$$
\begin{aligned}
& \dot{Z}=2 \dot{P} /|\dot{I}|^{2}=z_{a} z_{t} R_{D C}+\mathrm{j} \omega \int_{\Omega_{h}}\langle\dot{\mu}\rangle|\dot{H}|^{2} d \Omega /|\dot{I}|^{2} \\
& =z_{a} z_{t} R_{D C}+\mathrm{j} \omega \frac{\langle\dot{\mu}\rangle}{|\dot{I}|^{2}} \int_{0}^{b_{c}} \int_{0}^{h_{c}} \int_{0}^{l} \frac{b_{c}^{2} J^{2}}{b^{2}} y^{2} d x d y d z \\
& =z_{a} z_{t} R_{D C}+\mathrm{j} \omega\langle\dot{\mu}\rangle \frac{h_{c} b_{c} l z_{a}^{2} z_{t}^{2}}{3 b^{2}}
\end{aligned}
$$

\section{ACKNOWLEDGEMENT}

This work was supported by JSPS KAKENHI Grant Number JP1920541.

\section{REFERENCES}

[1] J. Gyselinck and P. Dular, "Frequency-domain homogenization of bundles of wires in 2-D magnetodynamic FE calculations," IEEE Trans. Magn., vol. 41, no. 5, pp. 1416-1419, May 2005.

[2] A. T. Phung, G. Meunier, O. Chadebec, X. Margueron, and J. P. Keradec, "High-frequency proximity losses determination for rectangular crosssection conductors," IEEE Trans. Magn., vol. 43, no. 4, pp. 1213-1216, Apr. 2007.

[3] H. Igarashi, "Semi-Analytical Approach for Finite-Element Analysis of Multi-Turn Coil Considering Skin and Proximity Effects," IEEE Trans. Magn., vol. 53, no. 1, pp. 1-7, Jan. 2017.

[4] Y. Sato and H. Igarashi, "Homogenization Method Based on Model Order Reduction for FE Analysis of Multi-Turn Coils," IEEE Trans. Magn., vol. 53, no. 6, pp. 1-4, 2017.

[5] Y. Sato and H. Igarashi, "Time-Domain Analysis of Soft Magnetic Composite Using Equivalent Circuit Obtained via Homogenization," IEEE Trans. Magn., vol. 53, no. 6, pp. 1-4, 2017.

[6] P. L. Dowell, "Effects of eddy currents in transformer windings," Proc. Inst. Electr. Eng., vol. 113, no. 8, p. 1387, Jun. 1966.

[7] D. Murthy-Bellur, N. Kondrath, and M. K. Kazimierczuk, "Transformer winding loss caused by skin and proximity effects including harmonics in pulse-width modulated DC-DC flyback converters for the continuous conduction mode," IET Power Electron., vol. 4, no. 4, p. 363, May 2011.

[8] S. Hiruma and H. Igarashi, "A study on orthogonal polynomials and Stieltjes continued fraction," Pap. Jt. Tech. Meet. Static App. Rotating Mach., vol. SA-18-013, pp. 1-8, 2018 (in Japanese), to be submitted to IEEE Trans. Magn.

[9] J. Pyrhönen, T. Jokinen, and V. Hrabovcová, Design of Rotating Electrical Machines, Second edi. Wiley, 2014.

[10] J. Lammeraner and M. Stafl, Eddy Currents. London, U.K.: ILIFFE Books, 1966. 
[11] P. Feldmann and R. W. Freund, "Efficient Linear Circuit Analysis by Padé Approximation via the Lanczos Process," IEEE Trans. Comput. Des. Integr. Circuits Syst., vol. 14, no. 5, pp. 639-649, 1995. 